\title{
Case Report: Hyperlordosis Secondary to Myasthenia Gravis Successfully Treated With Brace-wearing and Lordosis Exercises: A Case Report
}

\author{
Seyed Mani Mahdavi ${ }^{1}$ (D), Shayan Amiri ${ }^{2 *}$ (D), Farshad Safdari ${ }^{3}$ (i) \\ 1. Department of Orthopedic Spine Surgery, Rasoul-e-Akram Hospital, Iran University of Medical Sciences, Tehran, Iran. \\ 2. Department of Orthopedic Surgery, Rasoul-e-Akram Hospital, Iran University of Medical Sciences, Tehran, Iran. \\ 3. Bone Joint and Related Tissues Research Center, Shahid Beheshti University of Medical Sciences, Tehran, Iran.
}

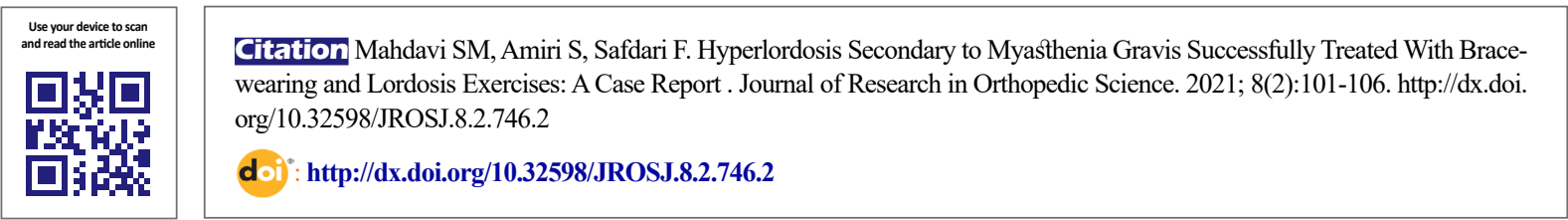

(c) (1) (5)

Article info:

Received: 16 Jan 2021

Revised: 25 Feb 2021

Accepted: 22 Mar 2021

Available Online: 01 May 2021

Keywords:

Myasthenia gravis,

Hyperlordosis, Spine deformity

\begin{abstract}
A B S T R A C T
Spinal deformities secondary to Myasthenia Gravis (MG) are rarely reported and mainly include scoliosis. In this study, we report a case of hyperlordosis with MG origin, which has not been reported earlier. The patient was a 12-year-old boy who underwent thymectomy at the age of three, and the disease was well-controlled afterward. One year ago, the patient presented with fatigue and severe low back pain associated with walking, while his parents noticed his buttocks jut out more than normal. The radiologic evaluation revealed a hyperlordosis of $100^{\circ}$. The patient was treated with a Milwaukee brace and lordosis exercises. One year after the treatment, an improvement of $27^{\circ}$ was observed in the hyperlordosis of the patient. While bracing does not typically treat a neuromuscular deformity, the present case reveals the value of bracing in the treatment of hyperlordosis with MG origin.
\end{abstract}

\section{Introduction}

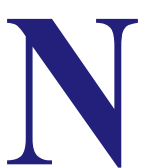

euromuscular spine deformity refers to spinal deformity secondary to various neuromuscular disorders, such as muscular dystrophy and cerebral palsy [1]. These deformities result from an imbalance between postural and muscular forces applied to the axial skeleton during the growth phase and are generally associated with severe curve progression [1]. Scoliosis with concomitant kyphosis is the most commonly reported neuromuscular spine deformity [2]. Hyperlordosis of the lumbar spine secondary to the neuromuscular origin is rarely reported [1]. Treatment of underlying neuromuscular disorder may prevent spinal deformity progression, while some treatments may accelerate it. Bracing typically does not treat a neuromuscular deformity, and surgical intervention is often necessary to manage the deformity in these patients [3].

\section{"Corresponding Author:}

Shayan Amiri, Orthopedic Surgery Resident.

Address: Department of Orthopedic Surgery, Rasoul-e-Akram Hospital, Iran University of Medical Sciences, Tehran, Iran.

Phone: +98 (912) 4578109

E-mail: amiri.shayan23@gmail.com 
Myasthenia Gravis (MG) is the most common autoimmune disease of neuromuscular origin characterized by activity-dependent muscle weakness. Life-threatening respiratory weakness is the most common MG symptom in nearly $15 \%$ of the patients [4]. Neuromuscular spine deformity secondary to $\mathrm{MG}$ has been rarely reported and mainly includes scoliosis $[5,6]$. To the best of our knowledge, hyperlordosis secondary to MG has not been reported in earlier investigations.

In this study, we report a case of a 12-year-old boy, which was presented with hyperlordosis secondary to MG and successfully treated with bracing and lordosisspecific exercises.

\section{Case Presentation}

A 12-year-old boy was referred to our orthopedic center with fatigue and severe low back pain associated with walking. In addition, the patient's parents were concerned about his appearance, as the patient's buttocks seemed to jut out more than normal. In history taking, it was turned out that the patient has a history of MG, which was diagnosed when he was three months old. The patient had experienced several rounds of pneumonia. Subsequently, the patient underwent thymectomy when he was three years old. Afterward, the disease was controlled with pyridostigmine and azathioprine and had no manifestation until the age of 12 years.

In clinical examination, fatigable muscle weakness was detected. Neurological examination, including reflexes, muscle strength, muscle tone, senses of touch and sight, coordination, and balance, were normal. Re- petitive nerve stimulation revealed poor ability to send a signal with fatigue. Anti-acetylcholine receptor antibodies were in the normal range $(<0.40 \mathrm{nmol} / \mathrm{L})$. Blocking antibody and modulating antibodies were in the normal range as well ( $<26 \%$ and $<45 \%$, respectively). Other laboratory indices such as Complete Blood Count (CBC), comprehensive metabolic panel, and liver function tests were normal. The electromyography revealed myogenic changes in the proximal upper and lower extremity. In radiologic evaluation, a lumbar hyperlordosis of $100^{\circ}$ (T12-S1) was detected (Figure 1). Consequently, the diagnosis of hyperlordosis secondary to MG was made.

We decided to treat the hyperlordosis with a standard Milwaukee brace and lordosis exercises. In this respect, the Milwaukee brace was custom-molded to the patient and administered 23 hours a day. Intra-abdominal pressure was retained more than usual to decrease the compressive loading of the spine. The patient was asked to stop bracing in case of having reflux or breathing difficulty. We applied the brace to provide a posterior pelvic tilting and anterior bending of the hip joints, thereby helping the correction of lordosis. The 1-hour out of the brace was spent doing exercises, which included strengthening, stretching, endurance, and resistance exercise.

Six months after the bracing, hyperlordosis was significantly improved so that the radiologic evaluation revealed $35^{\circ}$ correction in hyperlordosis in the plain radiography, which was obtained inside the brace (Figure 2). One year after the bracing, the hyperlordosis was further corrected so that a lordosis angle of $73^{\circ}$ was recorded without the brace (Figure 3). Considering the lordosis

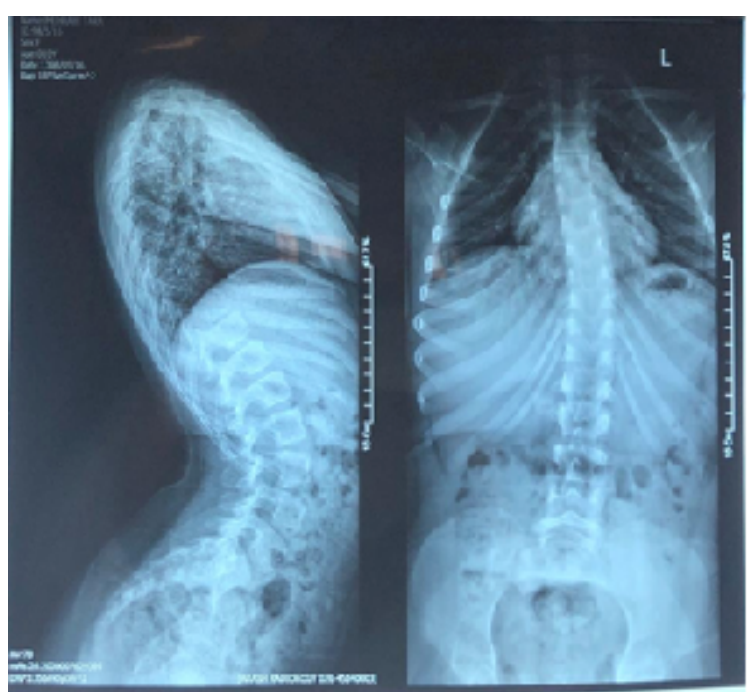

Figure 1. Plain radiographic before the treatment

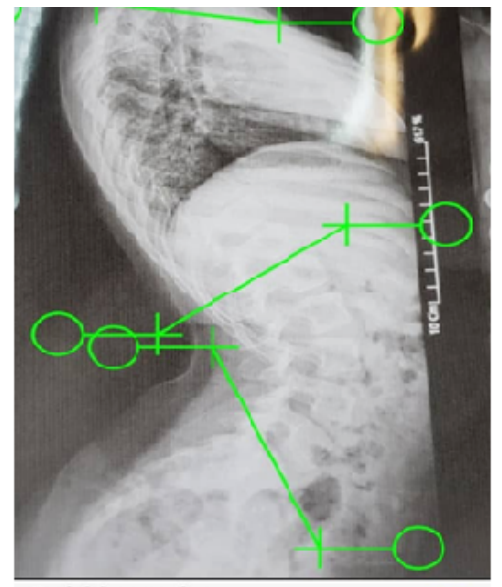

Cobb's angle:

highter- niddle $=41.3^{\circ}$

middle-lower $=99.9$ 

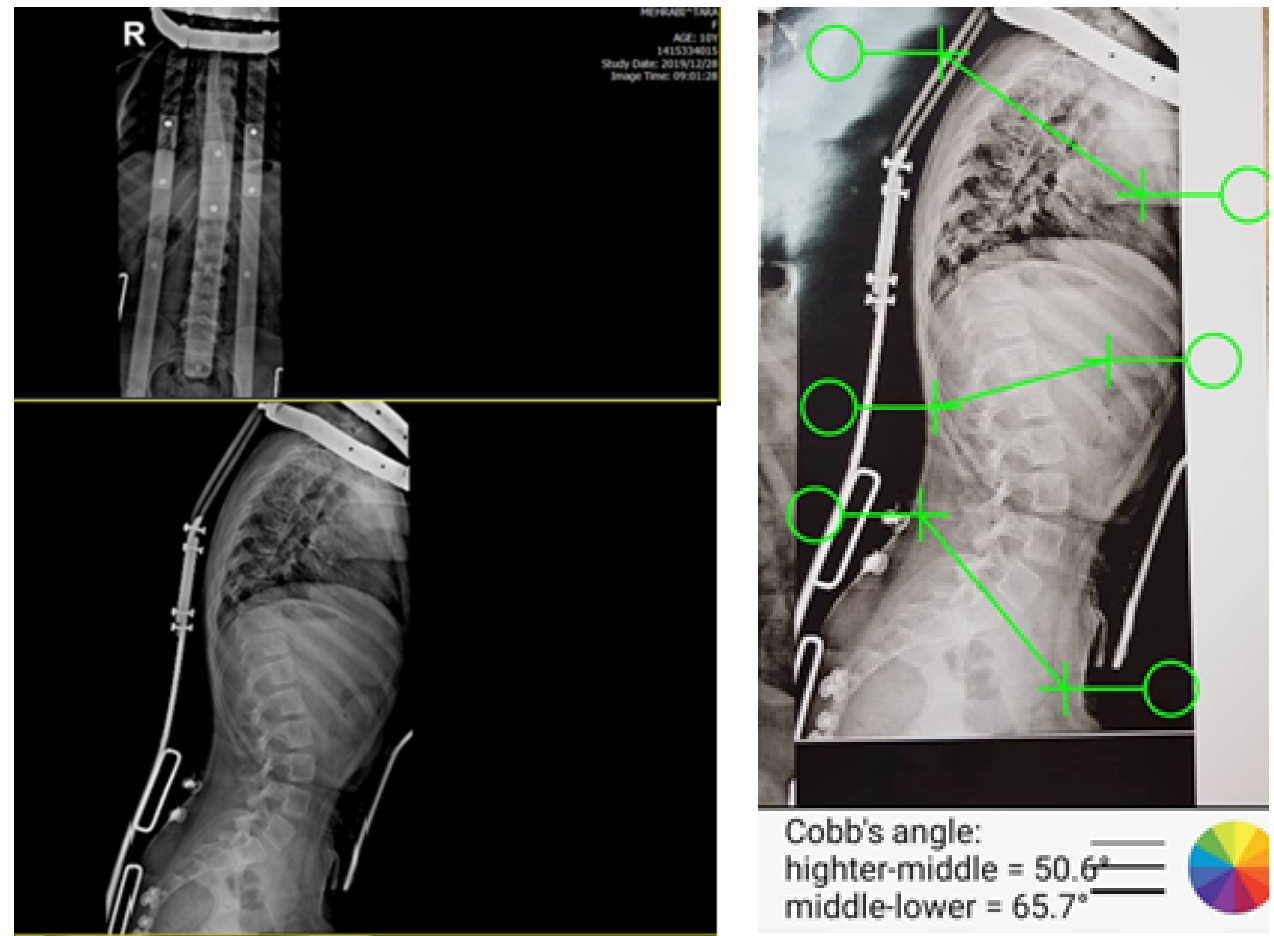

Figure 2. Plain radiographic 6 months after the treatment while using brace

Orthopedic Science

responsiveness to bracing and lordosis exercises, these modalities were continued for another year.

\section{Discussion}

Here, we reported a case of hyperlordosis secondary to the MG, which was occurred several years after thymectomy. While bracing does not typically prevent the progression of curves in neuromuscular spinal deformities, the hyperlordosis was significantly improved in this case following the application of Milwaukee brace and lordosis exercises.

Lumbar hyperlordosis of neuromuscular origin is limitedly reported in the literature. Vialle et al. [1] reported lumbar hyperlordosis of neuromuscular origin in 27 pa-

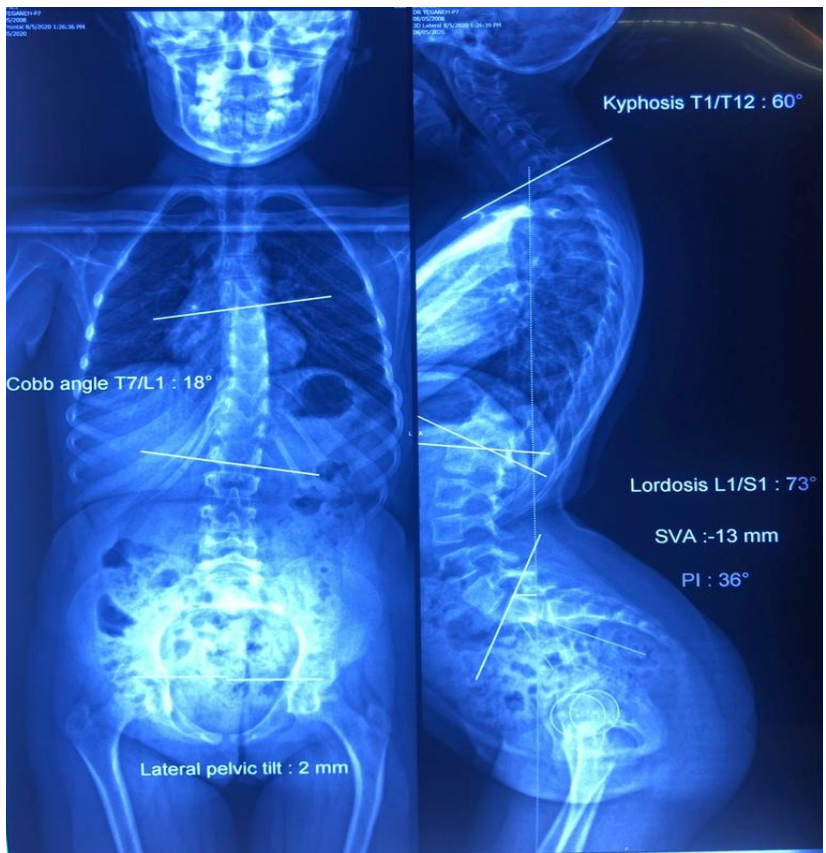

Figure 3. Plain radiographic 1 year after the treatment 
tients with sitting problems. The underlying neuromuscular disorder was cerebral palsy in 23 cases, congenital myopathy in 2 cases, lumbar meningocele in 1 case, and occipital meningocele in another case. All patients underwent surgical treatment, which resulted in significant improvement in hyperlordosis and sacral slope in 26 cases. The mean preoperative hyperlordosis and sacral slope of the patients were $102.6^{\circ}$ and $85.3^{\circ}$. At a mean follow-up of 5.3 years (range 1-15), mean hyperlordosis and sacral slope improved $35.3^{\circ}$ and $23.7^{\circ}$, respectively. The functional status of the patients was also improved with a comfortable sitting posture and good sagittal balance [1]. The hyperlordosis of the present case was $100^{\circ}$ before the treatment and $73^{\circ}$ one year after treatment. Accordingly, an improvement of $27^{\circ}$ was observed in hyperlordosis of the present following brace wearing and lordosis exercises, comparable to the improvements observed after the surgical treatment in the Vialle et al. study.

Spinal deformity in the context of MG is rare and is mainly presented in scoliosis form $[6,7]$. However, to the best of our knowledge, hyperlordosis secondary to MG has not been reported in the literature, and therefore, the optimal treatment of this condition is unknown. Olafsson and Al-Dabbagh reviewed the outcomes of 90 patients with various neuromuscular diseases and a progressive spinal deformity that were treated with a prefabricated Boston-type underarm corrective brace. On average, 3.1 years (range, 1-5.5 years) after bracing, the treatment was successful in 23 patients, meaning a $<10^{\circ}$ curve progression during the observation period and good compliance with bracing. The bracing was only successful in ambulating patients with muscle hypotonia and short thoracolumbar/lumbar curves $\left(<40^{\circ}\right)$ and non-ambulating cases with spastic short lumbar curves. They concluded that these types of neuromuscular spinal deformities might be the only ones that respond to bracing treatment [8]. The present case suggests that bracing could be an adequate treatment for spinal deformities with MG origin if the disease is well-controlled by thymectomy and medication.

It should be noted that the risk of intraoperative and postoperative complications is higher in neuromuscular spinal deformities when compared with idiopathic spinal deformities [9]. Rumalla et al. reviewed 2154 spinal surgeries, which were performed for patients with neuromuscular scoliosis, and reported an overall complication rate of $40.1 \%$ [10]. Turturro et al. reviewed the outcome of surgery in a consecutive series of 185 patients with neuromuscular scoliosis over 30 years and reported a $30 \%$ rate of adverse events [11]. Considering the high rate of complications following the surgical treatment of neuromuscular spinal deformities, brace-wearing treatment contains a critical value in these patients. However, several aspects of bracing need optimization. It is unclear how long bracing should be applied for the patients. In addition, it is not known whether the correction remains stable or the deformity recurs after bracing. Therefore, further studies are required to specify the value of brace-wearing in neuromuscular spinal deformities secondary to MG.

\section{Conclusion}

Neuromuscular hyperlordosis could be a late consequence of MG. While bracing does not typically prevent the progression of curves in neuromuscular spinal deformities, the hyperlordosis was significantly improved following the application of brace and lordosis exercises in the present case. This observation reveals the value of bracewearing in hyperlordosis secondary to $\mathrm{MG}$ in patients in whom the MG symptoms are adequately controlled.

\section{Ethical Considerations}

\section{Compliance with ethical guidelines}

This study was approved by the Review Board of Department of Orthopedics Surgery, Hazrate Rasool Akram Hospital, Iran University of Medical Sciences, Tehran. This study also observed the Helsinki Declaration and its later amendments.

\section{Funding}

This research did not receive any specific grant from funding agencies in public, commercial, or non-profit sectors.

\section{Authors' contributions}

All authors equally contributed to preparing this article.

\section{Conflict of interest}

The authors declared no conflict of interests.

\section{Acknowledgments}

This work was done in the Spine Clinic of Rasool Akram Hospital. We should appreciate the patient and his parents for helping in collecting the data. 


\section{References}

[1] Vialle R, Khouri N, Glorion C, Lechevallier J, Morin C. Lumbar hyperlordosis of neuromuscular origin: Pathophysiology and surgical strategy for correction. Int Orthop. 2007; 31:513-23. [DOI:10.1007/s00264-006-0218-4]

[2] Ferguson RL, Allen BL. Considerations in the treatment of cerebral palsy patients with spinal deformities. Orthop Clin North Am. 1988; 19(2):419-25. [DOI:10.1016/S00305898(20)30321-7]

[3] Murphy RF, Mooney 3rd JF. Current concepts in neuromuscular scoliosis. Curr Rev Musculoskelet Med. 2019; 12:220-7. [DOI:10.1007/s12178-019-09552-8]

[4] Statland JM, Ciafaloni E. Myasthenia gravis: Five new things. Neurol Clin Pract. 2013; 3(2):126-33. [DOI:10.1212/ CPJ.0b013e31828d9fec]

[5] Boghen D, Leclaire R, Fowles JV. Scoliosis and myasthenia gravis. A case report. Acta Orthop Belg. 1975; 41(6):660-2. [PMID]

[6] Hirai N, Hida S, Yo M, Naito M, Kawanami S. Degenerative scoliosis associated with myasthenia gravis. Orthop Traumatol. 2000; 49(4):1166-8. [DOI:10.5035/nishiseisai.49.1166]

[7] Dickson RA. Scoliosis in the community. Br Med J (Clin Res Ed). 1983; 286(6375):615-8. [DOI:10.1136/bmj.286.6365.615] [PMID] [PMCID]

[8] Olafsson Y, Saraste H, Al-Dabbagh Z. Brace treatment in neuromuscular spine deformity. J Pediatr Orthop. 1999; 19(3):376-9. [DOI:10.1097/01241398-199905000-00017]

[9] Reames DL, Smith JS, Fu KM, Polly Jr DW, Ames CP, Berven $\mathrm{SH}$, et al. Complications in the surgical treatment of 19,360 cases of pediatric scoliosis: A review of the scoliosis research society morbidity and mortality database. Spine. 2011; 36(18):1484-91. [DOI:10.1097/ BRS.0b013e3181f3a326]

[10] Rumalla K, Yarbrough CK, Pugely AJ, Koester L, Dorward IG. Spinal fusion for pediatric neuromuscular scoliosis: National trends, complications, and in-hospital outcomes. J Neurosurg Spine. 2016; 25(4):500-8. [DOI:10.3171/2016.2.SPINE151377]

[11] Turturro F, Montanaro A, Calderaro C, Labianca L, Di Sanzo V, Ferretti A. Rate of complications due to neuromuscular scoliosis spine surgery in a 30-years consecutive series. Eur Spine J. 2017; 26:539-45. [DOI:10.1007/s00586-017-5034-6] 
This Page Intentionally Left Blank 